\title{
Características del hábitat del Manatí antillano (Trichechus manatus manatus) en sistemas fluvio- lagunares del sur del Golfo de México
}

\author{
Darwin Jiménez-Domínguez ${ }^{1}$ y León David Olivera-Gómez ${ }^{1 *}$
}

Introduction: Studying a species habitat is a tool for researchers and stake holders to find key ecological characteristics to be used in management actions. Freshwater systems comprises most of the habitat of Antillean manatees, however the habitat features of these systems are still poorly understood. The objective of this study was to explore habitat variables related most to the use of freshwater systems by the Antillean manatees on the low basin of Usumacinta river.

Methods: We built generalized linear models using data on presence/absence and from sightings, achieved by direct and indirect sources on 62 navigations, between March 2006 and November 2007. For the models we used four types of variables: biological, physical, anthropic and from landscape, all previously reported as important in the habitat use of Antillean manatee.

Results: In general, the area (Fig. 1) had warm and shallow waters (2 to 4 m) with low transparency; waters are mostly lentic except for secondary and main rivers; vegetation was mostly of bank grasses and shrubs and boat traffic was medium. From the 19 initial habitat variables evaluated here, the best presence-absence model included transparency, distance to confluence and submerged vegetation (tables 1 and 2). The best sightings model was built with current and distance to streams (tables 1 and 2).

Discussion and conclusions: On the study area, manatees use deeper lentic environments near to feeding areas, like streams or confluences, where they find shelter. Conectivity is important in the habitat of manatees, they could be using shallow lakes to feeding in times of the day not comprised in this study, but then traveled to deeper watercourses using confluences. Manatees have also been reported to use these environments in Central and South America. Along freshwater systems associated to Usumacinta River, manatee is still abundant, confirming this region as a key area for manatee conservation in México. Results of this study had been use to classify the region and to prioritize research and conservation actions in a landscape scale.

Key words: freshwater systems, Generalized Linear Models, habitat, México, Sirenians, Usumacinta River.

\section{Resumen}

Conocer el hábitat de una especie ayuda a identificar características ecológicas clave para su manejo. Los sistemas fluvio-lagunares abarcan gran parte del hábitat del manatí Antillano. A pesar de esto, las características de su hábitat en estos sistemas aún son poco conocidas. El objetivo del presente trabajo fue explorar variables del hábitat relacionadas

\footnotetext{
'División Académica de Ciencias Biológicas, Universidad Juárez Autónoma de Tabasco. Carretera Villahermosa-Cárdenas Km. 0.5 S/N entronque a Bosques de Saloya. Villahermosa 86039, Tabasco, México. E-mail: leon_olivera@yahoo.com. mx (LDOG), darwin.oikos@gmail.com (DJD).

"Corresponding author
} 
con el uso de sistemas fluvio-lagunares por el manatí Antillano en la cuenca baja del río Usumacinta. Se construyeron modelos lineales generalizados utilizando datos de presencia-ausencia y número de avistamientos en puntos a lo largo de 62 navegaciones entre marzo de 2006 y noviembre de 2007. Para los modelos se utilizaron variables biológicas, físicas, antrópicas y de paisaje, reportadas previamente como importantes en el uso de hábitat del manatí Antillano. En general, el área de estudio comprende aguas tropicales, someras (2 a 4 m), con baja transparencia y en su mayoría lénticas.

La vegetación dominante son pastos y arbustos de orilla y el tráfico de embarcaciones es de mediana intensidad. El mejor modelo de presencia/ausencia se construyó con transparencia, distancia a confluencias y vegetación sumergida; el mejor modelo de avistamientos se construyó con corriente y distancia a arroyos. Los manatíes utilizan ambientes lénticos cercanos a áreas de alimentación, con mayor profundidad, como arroyos y confluencias, donde encuentran refugio. La conectividad es un elemento importante del hábitat del manatí Antillano. Los resultados servirán para clasificar la región y priorizar esfuerzos de investigación y conservación, a escala de paisaje.

Palabras clave: hábitat, México, Modelos Lineares Generalizados, Río Usumacinta, Sirenios.

Introducción

El hábitat del manatí Antillano (Trichechus manatus manatus) se compone de una gran diversidad de ambientes, tales como lagunas continentales, ríos, arroyos, zonas inundables, zonas costeras y estuarios (Lefebvre et al. 2001). En estos ambientes destacan como variables significativas del hábitat la cercanía a fuentes de agua dulce (OliveraGómez y Mellink 2005), la influencia de la disponibilidad de alimento (Axis-Arroyo et al. 1998), la preferencia de zonas someras cercanas a otras más profundas (OliveraGómez y Mellink 2005; Morales-Vela et al. 2000) y zonas con baja o nula actividad antrópica (Ortega 1997).

Algunas de las características más importantes del hábitat del manatí en sistemas de agua dulce, son la presencia de cursos de agua interconectados, con abundante vegetación acuática, aguas tropicales y transparentes y alta cobertura arbórea en las riberas, notándose una preferencia hacia el uso de lagunas amplias (Bermúdez et al. 2004; Jiménez 2005). Jiménez (2005) propuso un modelo general de las características del hábitat para cursos de agua dulce en Centroamérica; sin embargo, esta área no es representativa de la gran complejidad de los sistemas tropicales de agua dulce donde se encuentra al manatí.

En el sur del Golfo de México, las cuencas bajas de los ríos Grijalva y Usumacinta forman una planicie de inundación extensa y compleja, donde los manatíes se consideran abundantes (Colmenero y Hoz 1986; Álvarez et al. 1988). Esta extensa región brinda una oportunidad para seguir definiendo el hábitat del manatí, debido a su complejidad y dinámica hidrológica, la cual puede influir significativamente en el uso de hábitat por el manatí.

El objetivo de este estudio fue caracterizar y determinar la importancia de variables del hábitat relacionadas con el uso por parte del manatí de los sistemas fluvio-lagunares del sur del Golfo de México, principalmente en la cuenca baja del río Usumacinta, 


\section{Material}

con el fin de afinar la información sobre el hábitat de esta especie en sistemas de agua dulce. Esta información permitirá conocer mejor a los manatíes en una de sus zonas prioritarias, contar con mayores criterios que apoyen los planes de conservación y manejo y optimizar recursos y esfuerzos de investigaciones futuras.

\section{y Métodos}

Se trabajó en la cuenca baja del río Usumacinta y parte del río Grijalva, en el sur del Golfo de México (18 $36^{\prime}$ a $17^{\circ} 29^{\prime} \mathrm{N},-91^{\circ} 11^{\prime}$ a $-92^{\circ} 46^{\prime} \mathrm{W}$; Fig. 1), en la provincia fisiográfica de la planicie costera del Golfo de México (Toledo 2002). El clima en la región es cálido-húmedo, con una temperatura media anual de $27^{\circ} \mathrm{C}$ y una precipitación anual de 2,700 mm. La temporada de lluvias ocurre de finales de junio a febrero, y la temporada de sequías va de marzo a junio. En la estación lluviosa las tormentas y ciclones tropicales generan lluvias intensas que provocan desbordamiento de ríos y la consecuente inundación de zonas adyacentes. Existen alrededor de 12,312 Ha de lagunas permanentes y 9,256 Ha de lagunas temporales en la región de estudio (Rodríguez 2002). Estos cuerpos de agua se encuentran conectados por arroyos y ríos secundarios, afluentes de los ríos Usumacinta y Grijalva.

Figura 1. Área de estudio situada en el sur del Golfo de México, sobre las cuencas bajas de los ríos Usumacinta y Grijalva. Los círculos negros son localidades donde se realizaron los registros de ocurrencia de manatíes y de las variables del hábitat evaluadas.

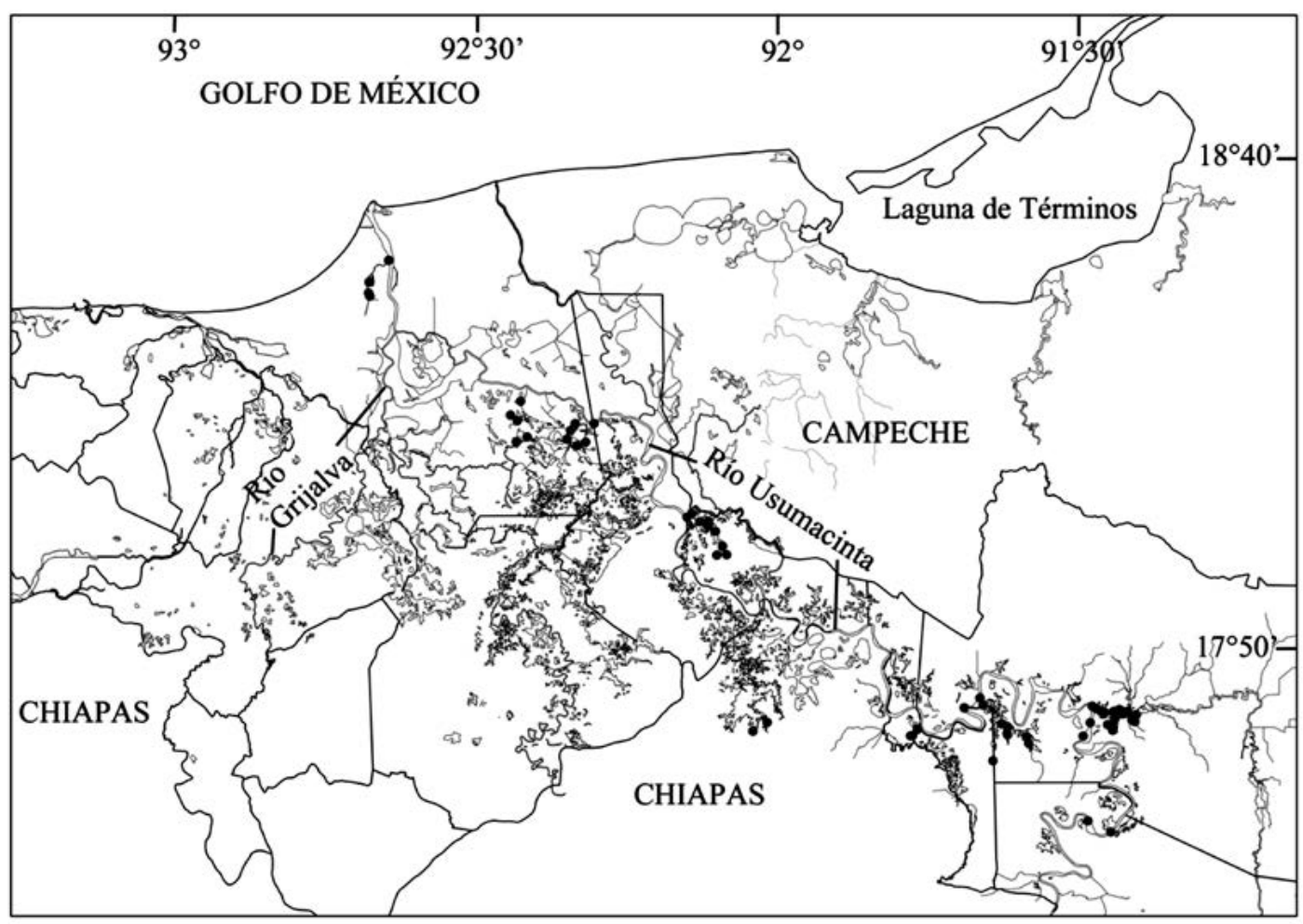

En esta cuenca existe la mayor diversidad de plantas asociadas a ambientes acuáticos de Mesoamérica, alrededor de 158 especies, que cubren aproximadamente 323,140 Ha en la región (Novelo 2006). Mientras que la vegetación arbustiva y arbórea que integran la selva baja y mediana inundable cubren aproximadamente 52,600 Ha (Romero et al. 2000; Anónimo 2006; Novelo 2006).

Entre marzo de 2006 y octubre de 2007, se realizaron 62 navegaciones de $1 \mathrm{~km}$ de largo cada una, para caracterizar 19 variables del hábitat que pudieran estar relacionadas 
con el uso que el manatí hace de los sistemas fluvio lagunares (SFL). Se usó una lancha de fibra de vidrio de $6 \mathrm{~m}$ de eslora con motor fuera de borda de 15 a 40 HP. Las navegaciones se realizaron sobre cinco diferentes tipos de ambientes, seleccionados de acuerdo con los sitios donde se reporta la presencia o ausencia de la especie en el área de estudio (Colmenero y Hoz 1986; Arriaga y Contreras 1993) y de informes obtenidos en entrevistas a pobladores de la región (Guzmán-Nieto 2008). Los ambientes acuáticos recorridos fueron: a) el río principal, que se caracteriza por tener velocidad de corriente alta, un ancho mayor a $100 \mathrm{~m}$ y profundidad promedio mayor a $5 \mathrm{~m}$; b) ríos secundarios, tributarios de ancho menor a $100 \mathrm{~m}$ y conectados al río principal; c) arroyos: cursos de agua (incluyendo canales artificiales) de ancho menor a $50 \mathrm{~m}$, que conectan lagunas con ríos secundarios; d) lagunas, cuerpos de agua extensos generalmente conectados a los ríos por medio de arroyos; e) confluencias, áreas de intersección de cuerpos de agua, generalmente lugares donde vierten sus aguas los ríos secundarios y arroyos hacia el río principal.

Se navegó una vez por sitio a una velocidad promedio de $10 \mathrm{~km} / \mathrm{h}$, y a una distancia de $20 \mathrm{~m}$, paralelo a la ribera de los cuerpos de agua. En caso de arroyos estrechos, éstos se recorrieron por el centro. La tripulación consistió de tres a cuatro observadores con experiencia en el reconocimiento de rastros de manatíes.

Variables del hábitat. Se consideraron cuatro tipos de variables ambientales: biológicas, físicas, antrópicas y de paisaje, reconocidas como determinantes en el uso del hábitat del manatí Antillano (Axis-Arroyo et al. 1998; Bermúdez et al. 2004; Jiménez 2005; Olivera-Gómez y Mellink 2005). Como variable biológica se consideró: la cobertura de vegetación, relacionada con la disponibilidad de alimento. Esta cobertura se subdividió en cuatro tipos: vegetación de ribera (VR), que son pastos, especies arbustivas y arbóreas que se encuentran a orillas de los cuerpos de agua; vegetación flotante (VF), plantas que flotan libremente sobre la superficie del agua y sólo sus raíces se encuentran sumergidas; vegetación emergente (VE), definida como la vegetación enraizada en el fondo de cuerpos de agua y que gran parte de sus hojas y estructuras florales sobresalen del agua; vegetación sumergida (VS), es la vegetación que se encuentra enraizada en el fondo y que toda su estructura vegetal está sumergida. También se incluyó a la vegetación de ribera que queda sumergida durante la época de inundaciones, como algunos pastos y plantas herbáceas que toleran esta condición.

Se estimó la cobertura de cada tipo de vegetación mediante inspección visual, en puntos al azar en las navegaciones y en los sitios donde se avistaron animales o se reportó su presencia, clasificándolas en las siguientes categorías de cobertura: [1] 1-25 $\%$, [2] 26-50 \%, [3] 51-75 \% y del [4] 76-100 \%. Los tipos de vegetación se incluyeron en una variable compuesta denominada suma de vegetación (SV), calculada mediante la suma de cada valor por categoría asignado a cada tipo de vegetación dentro del segmento muestreado, la cual se exploró en el modelo de forma independiente a las otras variables de vegetación.

Las variables físicas medidas fueron: temperatura del agua (Tmp), y la profundidad a diferentes distancias de la orilla: 5 m (P5m), 10 m (P10m) y 15m (P15m), medidas con ayuda de una ecosonda con sensor de temperatura y profundidad (Humminbird ${ }^{\circledR} 387 \mathrm{C}$ SI). La transparencia del agua (Trs) a $10 \mathrm{~m}$ de la orilla con ayuda del disco de Secchi y 
la velocidad de la corriente (VC) mediante una tarjeta de deriva, asignando los valores obtenidos dentro de cinco categorías: [0] 0m/s, [1] 0.01-0.25 m/s, [2] 0.26-0.50 m/s, [3] $0.51-0.75 \mathrm{~m} / \mathrm{s}$ y [4] $0.76-1.00 \mathrm{~m} / \mathrm{s}$.

La variable antrópica que se consideró fue el tráfico de embarcaciones (Trf), siguiendo el método utilizado por Jiménez (2005) mediante entrevistas a pobladores locales y a observaciones en campo, clasificándolo de acuerdo a su intensidad en el sitio en alguna de las siguientes categorías: [1] tráfico alto: rutas habituales de transporte, [2] tráfico medio: rutas marginales y áreas de pesca habitual, [3] tráfico bajo: áreas inaccesibles a las embarcaciones con motor o raramente visitadas. El tráfico de embarcaciones varía según la época de año, por lo que se registró en cada muestreo para asociarlo con las observaciones de manatíes.

Respecto a las variables de paisaje, se evaluó la distancia a los distintos ambientes desde los sitios de muestreo, midiendo la distancia a un arroyo (DA), rio principal (DRP), rio secundario (DRS), laguna (DL) y confluencia (DC), así como el nivel de conectividad con otro tipo de ambiente (NC). También se midió el ancho del curso de agua (ACA) como una medida de la dimensión de los cuerpos de agua. El NC se avaluó en categorías, mediante el siguiente criterio: [1] sin conexión a otro tipo de ambiente dentro de un radio de $10 \mathrm{~km}$; [2] conexión con un tipo de ambiente en un radio de $10 \mathrm{~km}$; [3] conexión con dos tipos de ambientes dentro de un radio de 10 km; [4] conexión con tres tipos de ambiente en un radio de $10 \mathrm{~km}$; [5] conexión con 4 tipos de ambientes dentro de un radio de $10 \mathrm{~km}$. Estas variables se estimaron utilizando ortofotos digitales escala 1:75,000 del año 2000 procesadas en el sistema de información geográfica ArcWiew 3.3 (ESRI Inc., 2002). En caso de que el ancho de un cuerpo de agua fuera menor a 100 $\mathrm{m}$, este se medía in situ con ayuda de un medidor laser de distancias digital (TruPulse 200, Laser Tecnology, Inc).

Registro de uso por el manatí. En las navegaciones de muestreo de las características del hábitat, se registró información sobre la presencia o ausencia de manatíes y número de avistamientos (Fig.1). Para el registro de la presencia del manatí se utilizaron métodos de registro directo e indirecto. Los registros directos consistieron en avistamientos de manatíes obtenidos por el grupo de observadores en campo, apoyados además de una ecosonda (Humminbird ${ }^{\circ}$ 987c SI) con imagen de barrido lateral para ubicar manatíes que estuvieran sumergidos y no fueran visibles para los observadores (González-Socoloske et al. 2009; González-Socoloske y Olivera-Gómez 2012). Además, en un punto fijo a lo largo de los segmentos se realizaron observaciones por medio de barrido visual con ayuda de binoculares por espacio de 30 a 60 min con el fin de detectar la presencia de manatíes en el sitio, tomando nota del número de manatíes observados.

Se consideraron como evidencias indirectas del uso de los diferentes ambientes por el manatí los rasgos o indicios que comprobaran la presencia del manatí en el área, tales como heces y vegetación con signos de forrajeo (Castelblanco et al. 2009). Así mismo, se consideró como registro indirecto el reporte de avistamientos de habitantes de la región que desarrollan alguna actividad relacionada con los cuerpos de agua que fueran confirmados por al menos dos personas de manera independiente (Bermúdez et al. 2004; Jiménez 2005). 
Análisis estadístico. Se realizó un análisis exploratorio construyendo una matriz de correlaciones de Spearman entre pares de variables consideradas, para excluir variables correlacionadas. Para explorar la importancia de las variables del hábitat en modelos de uso de los sistemas fluvio-lagunares por el manatí, se construyeron modelos lineales generalizados (MLG), los cuales son extensiones matemáticas de modelos lineales, que utilizan una función de enlace para inducir linealidad entre la variable de respuesta y las variables predictoras, que incorporan la variación no constante directamente en el análisis y restringen la respuesta dentro de un rango especifico (Redfern et al. 2006).

Estos modelos han sido utilizados anteriormente para modelar el uso de hábitat de manatíes en Centroamérica y el Caribe (Jiménez 2005; Olivera-Gómez y Mellink 2005), también se han usado para evaluar el uso de hábitat y distribución de otros mamíferos acuáticos como pinnípedos y cetáceos con fines de conservación y manejo (Redfern et al. 2006). Los MLG tienen la habilidad de manejar una gran clase de distribuciones de probabilidad para la variable de respuesta, tales como la distribución normal, binomial, Poisson, binomial negativa o gama, muchas de las cuales ajustan mejor las estructuras de error no normal de los datos ecológicos, las cuales pueden ser pobremente representadas por la clásica distribución Gausiana (Guisan et al. 2002; Jiménez 2005; Olivera-Gómez y Mellink 2005).

Los datos bivariados de presencia-ausencia se modelaron con una Regresión logística, mientras que para los datos de conteos se utilizó la Regresión de Poisson. Se evaluaron distintos modelos con combinaciones de las variables del hábitat mediante el método de selección por pasos (stepwise) hacia delante y hacia atrás, decidiendo mantener o excluir alguna variable específica mediante el contraste de la verosimilitud. Aunado a esto, para elegir los mejores modelos se utilizó el peso del Criterio de Información de Akaike corregido para pequeñas muestras (AICc), el cuál es un criterio de información teórica que nos permite identificar los modelos de máxima parsimonia con suficientes parámetros para describir la estructura de los datos sin sobreparametrización y pérdida de precisión (Burnham y Anderson 2002). Los análisis se realizaron con el programa SPSS 15.0 (SPSS Inc., 2006. Chicago, III, USA).

Variables del hábitat. De manera general, el área presenta aguas cálidas, con una baja transparencia y con profundidades promedio de dos a cuatro metros. La velocidad de corriente prácticamente es nula en la mayoría de los cuerpos de agua, a excepción del río principal y ríos secundarios. El tipo de cobertura vegetal predominante es la vegetación de ribera, la cual cubre por lo menos un 75 \% de los sitios muestreados, mientras que el tráfico de embarcaciones fue de mediana intensidad.

La profundidad a diferentes distancias y las variables de vegetación presentaron correlaciones significativas $(P<0.05)$ por lo que en los modelos se utilizaron de manera independiente. La velocidad de corriente y la distancia al río principal estuvieron correlacionadas positiva o negativamente con la mayoría de las otras variables, por lo que no se modelaron en compañía de las otras variables. 
Modelo de presencia/ausencia. Del universo de variables de hábitat evaluadas en este estudio, en los mejores modelos logísticos figuraron variables biológicas, físicas y de paisaje, quedando fuera la variable de tipo antrópico (Tabla 1). El mejor modelo seleccionado incluyó tres variables: Transparencia del agua, Distancia a confluencias y Cobertura de vegetación sumergida (Tabla 2). Al remover cualquiera de las variables incluidas, se produce un cambio significativo en el modelo final (Transparencia $P=0.041$, Distancia a confluencias $P=0.001$ y Vegetación sumergida $P=0.004$ ), lo que respaldó su permanencia en el modelo final. Con este modelo se clasificaron correctamente el $82.3 \%$ de los datos originales.

Modelo de avistamientos. En los mejores modelos de regresión de Poisson, las variables predictoras que se incluyeron correspondieron una variable física y una de paisaje (Tablas 1 y 2). Los modelos aquí generados sugieren una tendencia de los manatíes hacia el uso de ambientes con poca o nula corriente, cercanos a arroyos profundos. Adicionalmente, la presencia de vegetación y la cercanía a las confluencias resultaron ser variables importantes entre los mejores modelos alternativos (Tabla 1).

El modelo final presentó un buen ajuste, además se observó un efecto significativo al remover cualquiera de las variables del modelo (velocidad de corriente $P=0.001$, distancia a arroyos $P<0.001$ ), por lo que la inclusión de éstas mejora sensiblemente la verosimilitud del modelo.

Tabla 1. Contraste de los mejores modelos del uso de hábitat por el manatí para datos de presenciaausencia (regresión logística) y avistamientos (regresión de Poisson) en función de las variables del hábitat evaluadas en los sistemas fluvio lagunares del río Usumacinta. Para cada modelo se incluye el número de parámetros ( $k$ ), la devianza explicada del modelo $(-2 \log$ L), el Criterio de Información de Akaike ajustado para muestras pequeñas (AICC), la diferencia en AICc entre cada modelo propuesto y el modelo con el valor más bajo de $\mathrm{AICc}(\triangle \mathrm{AICc})$ y los pesos ponderados de Akaike (AICc w).

\begin{tabular}{lccccc}
\hline & $\mathrm{k}$ & -2 Log L & AICC & $\Delta$ AICC & AICc w \\
& & & & & \\
\hline & & & & & \\
Modelos de presencia/ausencia & & & & & \\
Trns+DC+VS & 5 & 42.84 & 53.91 & 0.00 & 0.36 \\
DC+VS+NC+Trns & 8 & 35.84 & 54.55 & 0.64 & 0.26 \\
DC+VC & 4 & 46.56 & 55.26 & 1.35 & 0.18 \\
DC+VS+NC+Trns & 9 & 34.89 & 56.35 & 2.44 & 0.11 \\
DC+VS & 7 & 41.52 & 57.60 & 3.69 & 0.06 \\
& & & & & \\
Modelos de Avistamientos & 6 & 218.50 & 232.03 & 0.00 & 0.94 \\
VCr+DA & 6 & 224.90 & 238.43 & 6.40 & 0.04 \\
VCr+DL & 6 & 226.30 & 240.58 & 8.56 & 0.01 \\
VCr+Trns & 12 & 210.72 & 241.09 & 9.07 & 0.01 \\
VCr+SV & & & & & \\
& & & & & \\
\hline
\end{tabular}

* DC, Distancia a confluencias; VS, Vegetación sumergida; Trns, Transparencia; NC, Nivel de Conectividad; P5, profundidad a $5 \mathrm{~m}$ de la orilla; VCr, Velocidad de corriente; DA, Distancia a arroyos; DL, Distancia a Lagunas; SV, Suma de vegetación.

\section{Discusión}

Las poblaciones de manatí Antillano en todo su rango de distribución se consideran reductos de lo que en el pasado fueron poblaciones más abundantes, debido principalmente a la cacería de la que fueron objeto (Lefebvre et al. 2001), por lo que algunos sitios pueden ser hábitat para el manatí aunque no sean ocupados actualmente. El manatí se encuentra ampliamente distribuido en el área de estudio (Colmenero y 
Hoz 1986; Arriaga y Contreras 1993) por lo que consideramos que los resultados de este trabajo pueden reflejar el uso del hábitat del manatí en sistemas dulceacuícolas tropicales.

Los sistemas fluvio-lagunares, como los que se presentan en el sureste de México y en muchos otros países donde se distribuye el manatí Antillano, son complejos y su descripción como hábitat de esta especie involucra la interacción de muchos factores de tipo físico, biológico, espacial y antrópico. De las diecinueve variables ambientales consideradas sólo seis de ellas (transparencia del agua, distancia a confluencias, porcentaje de vegetación sumergida, velocidad de corriente, distancia a arroyos y nivel de conectividad) se incluyeron en los mejores modelos de distribución del manatí en el área de estudio. La temperatura es una variable muy importante para explicar la distribución del manatí a escala mundial (Lefebvre et al. 2001). En la subespecie de Florida, que habita en las regiones más norteñas de la distribución de la especie, los manatíes se ven obligados a realizar movimientos invernales en búsqueda de aguas cálidas (Craig y Reynolds 2004). En sistemas tropicales la temperatura no parece ser un factor determinante (Bermúdez et al. 2004; Olivera-Gómez y Mellink 2005; Jiménez 2005; Montserrat et al. 2014).

La presencia de vegetación es una característica importante del hábitat del manatí, debido a que su dieta está dirigida principalmente a macrofitas (Marsh et al. 2011). En este estudio, la vegetación de ribera estuvo ampliamente disponible en toda el área de trabajo por lo que no se pudo definir su influencia como variable clasificatoria. La vegetación sumergida, sin embargo, sólo se encuentra en algunos sitios y esto pudo influir en el efecto significativo de esta variable en el modelo de presencia de manatíes, en especial en sitios con coberturas de 0 a 50\%. Esta vegetación sumergida, estuvo altamente correlacionada con los registros de vegetación emergente y con la suma de vegetación total, por lo que simplemente la forma de la distribución de los datos de la vegetación sumergida es lo que probablemente la hizo participar de manera significativa en el modelo final.

Algunos autores mencionan que la transparencia del agua no es una variable que influye de manera importante en el uso de hábitat del manatí (Hartman 1979, Auil 2004); sin embargo, Jiménez (2005), la reporta como significativa para modelar la presencia de manatíes en cursos de agua dulce. Aunque esta variable no resultó significativa en nuestro modelo, parece tener un efecto aditivo con las demás variables, ya que al excluirla del modelo, el ajuste se reduce significativamente. La transparencia del agua estuvo correlacionada con la vegetación emergente y sumergida, por lo que indirectamente se relaciona con la productividad de plantas hidrófilas debido a que favorece procesos físicos y geoquímicos que participan en el crecimiento de estas plantas.

Los sistemas fluvio-lagunares contienen diferentes ambientes (ríos, arroyos, lagunas). Las observaciones empíricas indican que las actividades de los manatíes varían entre estos ambientes (Colmenero 1984; Colmenero 1985). Se piensa que una mayor diversidad de ambientes cercanos interconectados puede llenar diferentes necesidades para un manatí y propiciar una mayor ocupación (Hartman 1979; Reynolds 1981). El nivel de conectividad (NC), que es la variable con la que estimamos el grado de conexión entre ambientes, no se incluyó significativamente en el modelo final de presencia ausencia, aunque formó parte de modelos alternativos que se acercaron en el ajuste al modelo 
final. Sin embargo, la distancia a confluencias (DC), es decir, donde se unen diferentes ambientes, fue una componente del modelo final y en el análisis exploratorio mostró correlación con el nivel de conectividad.

Los movimientos diurnos e intra-estacionales entre diferentes ambientes probablemente propician un mayor uso de las confluencias que los conectan. De acuerdo con otros autores y con nuestras observaciones en campo, los manatíes se mueven localmente de los ríos hacia las lagunas adyacentes y hacia zonas inundadas, influenciados por los pulsos de inundación (Colmenero 1984; Colmenero 1985), una vez que el nivel del agua comienza descender, los manatíes se desplazan hacia diferentes sitios como arroyos, al río principal, a sitios de confluencia o ambientes con mayor profundidad, donde encuentran refugio, aunque con menor accesibilidad y diversidad de plantas (Colmenero 1984; Colmenero 1985; Arriaga y Contreras 1993).

Una característica de las confluencias es que se forman depresiones en el fondo por efecto de la erosión de la corriente formándose lo que localmente se conocen como "pozas" donde los manatíes pueden refugiarse y descansar. Aunque en este estudio no se evaluó a estas zonas como microambientes, los pescadores locales hacen referencia constante a estos sitios como áreas con mayor probabilidad de avistamiento de manatíes. Por otro lado, las pozas, incluso en áreas abiertas, han demostrado ser un elemento del hábitat ampliamente utilizado por los manatíes en costas y bahías del Caribe (MoralesVela et al. 2000, Bacchus et al. 2007) y en Sudamérica (Castelblanco et al. 2009).

Con respecto a la velocidad de corriente, nuestros resultados coinciden con lo reportado por otros autores que evaluaron el uso de hábitat, ya que se ha observado una relación inversamente proporcional de esta variable con la presencia del manatí Antillano en ambientes de agua dulce de Centroamérica y la región de la Amazonia colombiana (Bermúdez et al. 2004; Jiménez 2005). La influencia de la corriente también se ha notado en estudios hechos en áreas abiertas estuarinas como la Bahía de Chetumal, en Quintana Roo, donde la protección contra el viento que genera corriente se relacionó positivamente con la presencia de manatíes (Axis-Arroyo et al. 1998). Adicionalmente, Gannon et al. (2007), sugieren que en Florida las hembras con cría prefieren ambientes que ofrezcan aguas tranquilas y con acceso a alimento. En este trabajo se observó frecuentemente hembras con cría en sitios con baja dinámica.

Para el modelo de avistamientos, la distancia a los arroyos, resultó importante, apoyando la hipótesis de que la disposición espacial de los diferentes tipos de ambientes influye en el uso del hábitat del manatí. Los arroyos conectan lagunas con ríos secundarios y principales, de hecho hubo correlación significativa entre la distancia a arroyos y la distancia a lagunas. Las lagunas propiamente, son un ambiente importante para los manatíes, debido a que típicamente presentan poca corriente, aunque probablemente, debido al tamaño de las unidades de muestreo y a que los arroyos son puntos obligados de acceso a las lagunas, su influencia no fue tan importante en la construcción de los modelos.

En trabajos donde se ha evaluado la distribución y uso de hábitat de fauna silvestre a través de un análisis multivariado basado en datos de avistamientos, se menciona la utilidad de este enfoque como herramienta para la toma de decisiones en la conservación y manejo de especies, principalmente de las que se tiene poca información, que son difíciles de estudiar en el campo y que son prioritarias para su conservación, como lo 
es en este caso el manatí (Palma et al. 1999; Jiménez 2005; Redfern et al. 2006). Es importante, sin embargo, evaluar las características del hábitat en regiones fisiográfica y ecológicamente diferentes, así como considerar explícitamente la dinámica temporal en los modelos, para cubrir mejor el espectro de condiciones que enfrenta una especie y que nos permitan tener generalizaciones para muchas otras áreas donde es más difícil realizar una evaluación.

\begin{tabular}{|c|c|c|c|c|}
\hline Variable* & Coeficiente & $\mathrm{Chi}^{2}$ de Wald & $\mathrm{gl}$ & $P$ \\
\hline \multicolumn{5}{|c|}{ Modelo de presencia/ausencia } \\
\hline DC & 0.000 & 3.528 & 1 & 0.060 \\
\hline VS (1-25\%) & 0.000 & 10.505 & 3 & 0.015 \\
\hline VS (26-50\%) & 1.869 & 4.955 & 1 & 0.026 \\
\hline VS (51-75\%) & 1.242 & 0.993 & 1 & 0.319 \\
\hline VS (76-100\%) & -0.776 & 0.377 & 1 & 0.539 \\
\hline Trns & -0.022 & 3.665 & 1 & 0.056 \\
\hline \multicolumn{5}{|c|}{ Modelo de avistamientos } \\
\hline DA & 0.000 & 10.138 & 1 & 0.001 \\
\hline $\mathrm{VC}(0 \mathrm{~m} / \mathrm{s})$ & 0.843 & 31.285 & 1 & 0.001 \\
\hline VC $(0.1-0.25 \mathrm{~m} / \mathrm{s})$ & 0.977 & 13.609 & 1 & 0.001 \\
\hline VC (0.26- $0.50 \mathrm{~m} / \mathrm{s})$ & 0.203 & 0.147 & 1 & 0.071 \\
\hline VC $(0.51-0.75 \mathrm{~m} / \mathrm{s})$ & 0.793 & 1.828 & 1 & 0.176 \\
\hline $\mathrm{VC}(0.76-1.00 \mathrm{~m} / \mathrm{s})$ & 0.505 & 0.249 & 1 & 0.618 \\
\hline
\end{tabular}

Tabla 2. Detalle de las variables incluidas en los modelos final de regresión logística para los datos de presenciaausencia y de regresión de Poisson para datos de avistamientos de manatíes en los sistemas fluvio lagunares del río Usumacinta.

Jiménez (2005) desarrolló modelos de hábitat para predecir la presencia del manatí en sistemas de agua dulce de Costa Rica, teniendo resultados positivos al generar modelos con un buen nivel de predicción de la presencia del manatí en la zona, aunque reconoce que sus observaciones aplican a cursos de agua cercanos a la costa. Axis-Arroyo et al. (1998), Olivera-Gómez y Mellink (2005) y Montserrat et al. (2014) evaluaron lagunas costeras con condiciones estuarinas y Reid et al. (2001) y Provancha y Hall (1991) trabajaron en zonas marinas costeras. Los complejos sistemas fluvio-lagunares, más alejados de la costa, evaluados en este estudio constituyen otro tipo de ambiente ecológico, muy característico de los sitios donde se distribuye la especie en México y Sudamérica.

La presencia de grandes ríos sobre planicies bajas de inundación es una condición importante que propicia el desarrollo de poblaciones importantes de manatíes como se nota al analizar la distribución de la especie (Lefebvre et al. 2001). Los ríos principales actúan como corredores para el desplazamiento de individuos, pero son los sistemas adyacentes los que soportan el establecimiento de las poblaciones. Adentro de estos sistemas la transparencia aumenta, la corriente disminuye y la vegetación se hace más densa y diversa (Hernández-Velázquez 2008). Los manatíes aprovechan la heterogeneidad que le proporciona la diversidad de ambientes dentro de los sistemas fluvio-lagunares (zonas inundables, lagunas, arroyos, ríos secundarios) como es notorio en los modelos obtenidos en este estudio.

Por esta razón es importante mantener la conectividad en estos sistemas, por una parte para no provocar el aislamiento de poblaciones y permitir el flujo génico (Sauders et al. 1991, García-Rodriguez et al. 1998; Bazzalo et al. 2008) y por el otro para asegurar la amplia gama de recursos y condiciones que le proporciona un sistema heterogéneo 
a los manatíes, favoreciendo el establecimiento de poblaciones saludables. Esto no es fácil, ya que se considera que aunque la disminución fuerte de las poblaciones de manatíes en México y en el resto de su rango de distribución se debe a la cacería intensiva a la que estuvo sujeta en el siglo pasado (Guichard et al. 2001; Anónimo 2002), la presión sobre el hábitat es ahora el principal problema de conservación (Anónimo 2002; SEMARNAT/CONANP 2010). El establecimiento de la industria de extracción de hidrocarburos, la deforestación y actividades agropecuarias, el desvío de los cauces de ríos y arroyos, desecación de lagunas, la colocación de redes a lo ancho de algunos ríos secundarios y arroyos, son problemas que enfrenta la especie donde se llevó a cabo el presente y muchos de ellos son comunes a otros sitios donde se distribuye la especie (González-Sokoloske et al. 2007).

La información generada provee mayores herramientas para el manejo del manatí en una escala de paisaje regional con un enfoque adaptativo, ayudando a la clasificación del hábitat en la región. Es necesario que la conservación y manejo de manatíes se incluya como parte activa en la conservación y manejo de los ecosistemas acuáticos de la cuenca baja de los Ríos Usumacinta y Grijalva, al ser un buen ejemplo de la aclimatación regional de muchas especies a los pulsos de inundación y un indicador de la degradación de los sistemas acuáticos.

\section{Agradecimientos}

Esta investigación fue financiada por el Fondo Sectorial de Investigación Ambiental SEMARNAT-2004-C01-00385. Agradecemos a los voluntarios del proyecto manatí-UJAT, pescadores de la región, a las autoridades locales del municipio de Balancán, Tabasco y al Instituto de Historia Natural de Chiapas por las facilidades y apoyo logístico otorgado para el trabajo de campo. En especial agradecemos a M. Hidalgo y a B. Morales por sus sugerencias al presente manuscrito.

\section{Literatura citada}

Átvarez, C, A. Aguayo, y L. Jhonson. 1988. Observaciones sobre el manatí Trichechus manatus en la región media del Usumacinta, Tabasco. Pp. 617-624 en Ecología de los ríos Usumacinta y Grijalva, Chávez-Lomelí, M. O., ed. Instituto Nacional de Investigación sobre Recursos Bióticos, División Regional Tabasco y Gobierno del Estado de Tabasco. Villahermosa, México.

ANónimo. 2002. Proyecto de conservación, recuperación y manejo del manatí, Trichechus manatus manatus, en México. Instituto Nacional de Ecología-Secretaría de Medio Ambiente y Recursos Naturales. Ciudad de México. Disponible en: http://www.semarnat.gob.mx/temas/gestionambiental/vidasilvestre/Documents/ Preps/Proyecto-Manati.pdf; última consulta: 26.IV.2014.

Anónimo. 2006. Anuario estadístico del estado de Tabasco. Instituto Nacional de Estadística, Geografía e Informática. Ciudad de México, México.

Arriaga, S., y W. Contreras. 1993. El manatí (Trichechus manatus) en Tabasco. Universidad Juárez Autónoma de Tabasco, División Académica de Ciencias Biológicas. Villahermosa, México. 
AuIL, N. E. 2004. Abundance and distribution trends of the West Indian manatee in the coastal zone of Belize: implications for conservation. Master in Science Thesis. Texas A\&M University. Nagle Hall, EE.UU.

Axis-Arroyo, J., B. Morales-Vela, D. Torruco-Gómez, y M. Vega-Cendejas. 1998. Variables asociadas con el uso de hábitat del manatí del Caribe (Trichechus manatus), en Quintana Roo, México (Mammalia). Revista de Biología Tropical 46:791-803.

Bacchus, M., S. G. Dunbar, y C. Self-Sullivan. 2007. Characterization of Resting Holes and Their Use by the Antillean Manatee (Trichechus manatus manatus) in the Drowned Cayes, Belize. Aquatic Mammals 35:62-71.

Bazzalo, M., P. Flores, y M. G. Pereira. 2008. Uso de hábitat y principales comportamientos del delfín gris (Sotalia guianensis, Van Bénéden, 1864) en la Bahía Norte, estado de Santa Catarina, Brasil. Mastozoología Neotropical 15:9-22.

Bermúdez, A. L., D. N., Castellblanco, y F. Trujillo. 2004. Patrones de presencia y uso diferencial del hábitat de Trichechus manatus manatus en el Río Orinoco dentro de la zona de influencia de Puerto Carreño, Vichada. Pp. 133-158 en Estudios de fauna silvestre en ecosistemas acuáticos en la Orinoquia Colombiana (Diazgranados, M., y F. Trujillo, eds). Instituto de Estudios Ambientales para el Desarrollo, Departamento de Ecología y desarrollo, Pontificia Universidad Javeriana. Bogotá, Colombia.

Burnham, K., y D. R. Anderson. 2002. Model selection and multimodel inference. Springer. New York, EE.UU.

Castelblanco, D. N., A. L. Bermúdez, A. V. Gómez, F. C. Weber, F. Trujlllo, y E. Zerda. 2009. Seasonality of habitat use, mortality and reproduction of the Vulnerable Antillean manatee Trichechus manatus manatus in the Orinoco River, Colombia: implications for conservation. Oryx, 43:235-242

Colmenero, L. C. 1984. Nuevos registros del manatí (Trichechus manatus) en el sureste de México. Anales del Instituto de Biología, Universidad Nacional Autónoma de México, Serie Zoología 54:243-254.

Colmenero, L. C. 1985. Aspectos de la ecología y comportamiento de una colonia de manatíes (Trichechus manatus) en el municipio de Emiliano Zapata, Tabasco. Anales del Instituto de Biología Universidad Nacional Autónoma de México, Serie Zoología 56: 589-602.

Colmenero L. C., y M. E. Hoz. 1986. Distribución de los manatíes, situación y su conservación en México. Anales del Instituto de Biología, Universidad Nacional Autónoma de México, Serie Zoología 56:955-1020.

Craig, B., y J. E. Reynolds. 2004. Determination of manatee population trends along the Atlantic coast of Florida using a bayesian approach with temperature-adjusted aerial survey data. Marine Mammals Science 20:386-400.

Gannon, J.G., K.M. Scolardi, J. E. Reynolds, J. K. Koelsch, y T. J. Kessenich. 2007. Habitat selection by manatees in Sarasota Bay, Florida. Marine Mammals Science 23:133-143.

García-Rodríguez, A. I., B. W. Bowen, D. Domning, A. A. Mignucci-Giannoni, M. Marmontel, R. A. Montoya-Ospina, B. Morales-Vela, M. Rudin, R. K. Bonde, y P. M. McGuire. 1998. Phylogeography of the West Indian Manatee (Trichechus manatus): How many populations and how many taxa? Molecular Ecology 7:1137-1149. 
González-Socoloske, D., L. D. Olivera-Gómez, y E. Quintana-Rizzo. 2007. Primer simposio para la biología y la conservación del manatí Antillano (Trichechus manatus manatus) en Mesoamérica. Mesoamericana 11:1-10.

González-Socoloske, D., L. D. Olivera-Gomez, y R. E. Ford. 2009. Detection of freeranging West Indian manatees (Trichechus manatus) with side-scan sonar. Endangered Species Research 8:249-257.

González-Socoloske, D., y L. D. Olivera-Gomez. 2012. Gentle Giants in Dark Waters: Using Side-Scan Sonar for Manatee Research. The Open Remote Sensing Journal 5:1-14.

Guichard, C, S. Ellis, Y. Matamoros y U. Seal. 2001. Análisis de la viabilidad poblacional y del hábitat del manatí en México. Informe, Conservation Breeding Specialist Group (SSC/IUCN) Apple Valley, Mn. Disponible en: www.cbsg.org/cbsg/ workshopsreports/23/manateephva 2002.pdf; última consulta 26/IV/2014.

Guisan, A., T. C. Edwards, y T. Hastie. 2002. Generalized linear and generalized additive models in studies of species distributions: setting the scene. Ecological Modelling 157:89-100.

Guzmán-Nieto, J. E. 2008. Conocimiento y apreciación del manatí y su relación con la calidad del hábitat en la cuenca del río Usumacinta. Tesis. Universidad Juárez Autónoma de Tabasco. Villahermosa, México.

Hartman, D. S. 1979. Ecology and behavior of the manatee (Trichechus manatus) in Florida. The American Society of Mammalogist Special Publication 5. Pittsburgh, EE. UU.

Hernández-Velázquez, J. A. 2008. Caracterización y evaluación del hábitat de la tortuga blanca Dermatemys mawii en la Reserva de la Biosfera Pantanos de Centla, Tabasco. Tesis. Universidad Juárez Autónoma de Tabasco. Villahermosa, México.

Jiménez, I. 2005. Development of predictive models to explain the distribution of the West Indian manatee Trichechus manatus in tropical watercourses. Biological Conservation 125:491-503.

Lefebvre, L., M. Marmontel, J. P. Reid, G. B. Rathbun, y D. P. Domning. 2001. Status and biogeography of the west Indian Manatee. Pp. 425-474.en Biogeography of the West Indies, patterns and perspectives (Woods, C. A., y F. E. Sergile, eds). CRC. Boca Raton, EE.UU.

Marsh, H., T. J. O'Shea, y J. E. Reynolds III. 2011. Ecology and Conservation of the Sirenia, Dugongs and Manatees. Conservation Biology 8, Cambridge University Press. New York, EE. UU.

Montserrat M., M. A. Vega-Cendejas y B. Morales-Vela. 2014. Ecological distribution of manatee in Bahía de la Ascensión, Mexico. Marine Mammal Science DOI: 10.1111/mms.12127.

Morales-Vela, B., L. D. Olivera-Gómez, J. Reynolds, y G. B. Rathbun. 2000. Distribution and habitat use by manatees (Trichechus manatus manatus) in Belize and Chetumal Bay, Mexico. Biological Conservation 95:67-75.

Novelo, L. A. 2006. Plantas acuáticas de la Reserva de la Biosfera Pantanos de Centla. Espacios Naturales y Desarrollo Sustentable, Ciudad de México, México.

Ouivera-Gómez, L. D., y E. MeluınK. 2005. Distribution of the Antillean manatee (Trichechus 
m. manatus) as a function of habitat characteristics in Bahia de Chetumal, Mexico. Biological Conservation 121:127-133.

Ortega, A. 1997. Uso de caletas y cenotes por el manatí del Caribe Trichechus manatus y el impacto del turismo en Quintana Roo, México. Tesis. Universidad Veracruzana. Xalapa, México.

Palma, L., P. BejA, y M. Rodríguez. 1999. The use of sighting data to analyze Iberian lynx habitat and distribution. Journal of Applied Ecology 36:812-824.

Provancha, J. A., y C. R. Hall. 1991. Observations of associations between seagrass beds and manatees in East Central Florida. Biological Sciences 54:87-98.

Redfern, J. V., M. C. Ferguson, M. A. Becker, K. D. Hyrenbach, C. Good, J. Barlow, K. Kaschner, M. F. Baumgartner, K. A. Forney, L. T. Balance, P. Fauchald, P. Halpin, T. Hamazaki, A.J. Pershing, S. Qian, A. Read, S. B. Reilly, L. Torres, y F. Werner. 2006. Techniques for cetacean-habitat modeling. Marine Ecology Progress Series 310:271-295.

Reid, J. P., D. Easton, y W. J. Kenworthy. 2001. Manatee and seagrass habitat characterization in Puerto Rico. Report to Department of the Navy, U.S Naval Station Roosevelt Roads and Sirenia Project, U. S. Geological Survey. Biological Resources Division.

ReYNOLDS, J. E. 1981. Aspects of the social behavior and herd structure of a semi-isolated colony of West Indian manatees, Trichechus manatus. Mammalian 45:431-451.

RodríGuEz, E. 2002. Las lagunas continentales de Tabasco. Universidad Juárez Autónoma de Tabasco. México. Villahermosa, México.

Romero, J. C., M. A. García, J. C. A. Bautista, y A. P. H. Pérez. 2000. Caracterización de la Reserva de la Biosfera Pantanos de Centla. Universidad y Ciencia. 15:15-28.

Sauders, D. A., R. J. Hobbs, y C. R. Margulis. 1991. Biological consequences of ecosystem fragmentation: a review. Conservation Biology 5:18-32.

SEMARNAT/CONANP. 2010. Programa de Acción para la Conservación de la Especie: Manatí (Trichechus manatus manatus). Compilado por: Olivera Gómez, L. D., A. Ortega-Argueta, B. Morales Vela, y L. C. Colmenero Rolón. Ciudad de México, México.

Toledo, A. 2002. Ríos, costas y mares, Hacia un análisis integrado de las regiones hidrológicas de México. INE-SEMARNAT. Ciudad de México, México.

Sometido: 20 de enero de 2014

Revisado: 15 de marzo de 2014

Aceptado: 20 de abril de 2014

Editor asociado: Sergio Ticul Álvarez-Castañeda

Diseño gráfico editorial: Gerardo Hernández 